\title{
Factor atención de salud y la adherencia al consumo de multimicronutrientes en madres con niños de 6-36 meses, que acuden al Centro de Salud Guadalupe, Juliaca - 2018
}

\author{
Health care factor and adherence of multimicronutrients consumption in mothers \\ with children from 6 to 36 who come to the health center Guadalupe, Juliaca -2018
}

\author{
Mayumy Mamani Tito' ${ }^{1}$, Yovana Jarata Hancco²
}

\begin{abstract}
RESUMEN
Objetivo la relación que existe entre el factor atención de salud y la adherencia al consumo de multimicronutrientes en madres con niños de 6 a 36 meses, Centro de Salud Guadalupe, Juliaca 2018. Metodología: Es un estudio de tipo descriptivo correlacional, de enfoque cuantitativo, diseño no experimental y de corte transversal. La muestra fue no probabilística de tipo intencional, utilizando los criterios de inclusión y exclusión, como muestra se consideró a 70 niños de 6 a 36 meses de edad. Instrumento utilizado para la recolección de datos fue el Test de factor atención de salud y adherencia al consumo de multimicronutrientes de Lazarte (2017). Resultados encontrados indican en relación a la atención de salud $60 \%$ percibieron una mala atención, 24.35 percibieron una buena atención y solo $15.7 \%$ percibieron una atención regular. En cuanto a la adherencia $72.9 \%$ no son adherentes y $27.1 \%$ fueron adherentes al consumo de multimicronutrientes. Conclusión: Por lo tanto, existe una relación directa entre el factor atención de salud y la adherencia al consumo de multimicronutrientes, con un p-valor de 0. 000.Conclusión existe relación significativa entre ambas variables.
\end{abstract}

Palabras clave: Atención de salud, adherencia, anemia, micronutrientes.

\begin{abstract}
Objective: the relationship between the health care factor and the adherence to multimicronutrients consumption in mothers with children from 6 to 36 months, Guadalupe Health Center, Juliaca - 2018. Metodology: It is a Correlational descriptive type study, quantitative approach, non-experimental and cross-sectional design. The sample was non-probabilistic of intentional type, using the inclusion and exclusion criteria, consisting of 70 children from 6 to 36 months of age. Instrument used for data collection was the Lazarte multi-micronutrient health care factor test and adherence to consumption (2017). The results: Found indicate in relation to health care $60 \%$ perceived poor attention, 24.35 perceived good care and only $15.7 \%$ perceived regular attention. Regarding adherence, $72.9 \%$ are not adherent and $27.1 \%$ were adherent to the consumption of multimicronutrients. Conclusion: there is a direct relationship between the health care factor and the adherence to multimicronutrient consumption, with a $p$-value of 0.000 . Conclusion there is a significant relationship between both variables.
\end{abstract}

Keywords: Health care, adherence, anemia, micronutrients. 


\section{INTRODUCCIÓN}

La Organización Mundial de la Salud (2003), define la adherencia al cumplimiento del tratamiento indicado, es decir, tomar la medicación de acuerdo con la dosificación del programa prescrito; y la persistencia, tomar la medicación a lo largo del tiempo. De otra manera, se califica la falta de adherencia como un problema mundial de gran magnitud, por ello es necesario dar importancia al problema para plantear estrategias que den solución. Por otro lado, el Ministerio de Salud (2016), indica a la calidad de la atención como el conjunto de características humanas, técnicas, científicas y materiales que debe tener la atención a los usuarios para satisfacer sus expectativas. Para esto se establece el "Manual de Estándares e Indicadores de Calidad para el Primer Nivel de Atención y Hospitales" y "Guía Técnica para la Evaluación de la Satisfacción del Usuario Externo en los Centros de atención de salud y de los Servicios Médicos de Apoyo", donde se han establecido los criterios mínimos de desempeño que permiten garantizar la calidad de las prestaciones de salud en los centros de todo el país.

El objetivo de este trabajo de investigación es determinar la relación que existe entre el factor atención de salud y la adherencia al consumo de Multimicronutrientes en madres con niños de 6 a 36 meses, Centro de Salud Guadalupe, se basó en las diferentes falencias que se observó en el centro de salud donde se ejecutó dicha investigación son las mismas que se presentan en cualquier hospital las cuales aún no se han podido mejorar, tales como: La infraestructura sin duda eso dificulta la atención de calidad para el usuario, personal limitado, esto sin duda hace mucho más cargado por el ambiente del personal que labora en este centro ya que los lleva a un alto nivel de estrés que termina siendo desquitado de cierta forma con los usuarios, haciendo de tal forma que no vuelvan a asistir a consulta perjudicando en si a los niños que tienen que recibir sus controles, exámenes y suplementos (Huerta, 2014).

\section{METODOLOGÍA}

Es un estudio de tipo descriptivo correlacional, de enfoque cuantitativo, diseño no experimental y de corte transversal.

\section{Instrumento}

Los instrumentos utilizados para la recolección de datos fue el Test de factor atención de salud y adherencia al consumo de multimicronutrientes creado por Lazarte (2017), los cuales fueron validados y fiabilizados por apreciación y juicio de expertos. El instrumento utilizado consta de dos partes, el primer instrumento lleva como título cuestionario atención de salud y el segundo Test de adherencia al consumo de multimicronutrientes, consta de preguntas de alternativas dicotómicas ( $\mathrm{SI}$ y NO), para medir la relación entre factor atención de salud y la adherencia del consumo de Multimicronutrientes.

\section{Participantes}

La muestra fue no probabilística de tipo intencional, utilizando los criterios de inclusión y exclusión, con una población total de 640 y un muestreo conformada por 70 madres con niños de 6 a 36 meses de edad.

\section{Análisis de datos}

Los datos que se obtuvieron fueron procesados en el programa de SPSS versión 23 para ser presentados en las tablas, posteriormente para luego realizar el análisis, para luego llegar a una interpretación, discusión delos resultadosyformular las diferentes conclusiones y recomendaciones del trabajo realizado. Procediendo a dar valor a las diferentes opciones de los ítems, verificado también que los datos estén completos. Finalmente se añadió una variable más factores relacionados a la no adherencia de los micronutrientes la cual se evaluó conforme a la medición del instrumento. Finalmente se realizaron cuadros con tablas de contingencia, las cuales cruzaron variables: Factor atención de salud y la adherencia al consumo de multimicronutrientes, para luego analizarlos mediante el estadístico Rho de Spearman y finalmente verificando las hipótesis planteadas.

\section{RESULTADOS Y DISCUSIÓN}

En la tabla 1 observamos que $60 \%$ de madres percibieron una mala atención de parte del personal de salud, haciendo que $58,6 \%$ sea no adherente, por consiguiente $24,3 \%$ percibieron atención buena, por lo tanto, hace que $17,1 \%$ sea adherente al consumo de multimicronutrientes. 
Tabla 1

Correlación entre el Factor Atención de Salud y a Adherencia del consumo de multimicronutrientes en madres con niños de 6 a 36 meses del Centro de Salud Guadalupe 2018.

\begin{tabular}{|c|c|c|c|c|c|c|}
\hline \multicolumn{7}{|c|}{ Adherencia del consumo de multimicronutrientes } \\
\hline \multirow{2}{*}{$\begin{array}{l}\text { Factor Atención } \\
\text { de Salud }\end{array}$} & \multicolumn{2}{|c|}{ No adherente } & \multicolumn{2}{|c|}{ Adherente } & \multicolumn{2}{|c|}{ Total } \\
\hline & $\mathrm{N}$ & $\%$ & $\mathrm{~N}$ & $\%$ & $\mathrm{~N}$ & $\%$ \\
\hline Malo & 41 & 58,6 & 1 & 1,4 & 42 & 60,0 \\
\hline Regular & 5 & 7,1 & 6 & 8,6 & 11 & 15,7 \\
\hline Bueno & 5 & 7,1 & 12 & 17,1 & 17 & 24,3 \\
\hline Total & 51 & 72,9 & 19 & 27,1 & 70 & 100,0 \\
\hline
\end{tabular}

Fuente: Encuesta aplicada a madres con niños de 6 a 36 meses.

En la tabla 2, se corrobora la hipótesis alterna, es decir que si existe correlación positiva moderada entre el factor atención de salud y la adherencia al consumo de multimicronutrientes, basándonos en los rangos de Spearman (0.60-0.80), lo cual indica el nivel crítico de es $<0.05$ es significativo, en este estudio se obtuvo un valor $p$ de 0,000 en la prueba estadística de Spearman

Tabla 2

Prueba de correlación de Rho de Spearman en el factor atención de salud y la adherencia al consumo de multimicronutrientes.

\begin{tabular}{|c|c|c|}
\hline $\begin{array}{l}\text { Factor atención de salud y la } \\
\text { adherencia al consumo de } \\
\text { Multimicronutrientes }\end{array}$ & Coeficiente de correlación & Valor $\mathrm{P}$ \\
\hline Factor atención de salud & 0,689 & 0,000 \\
\hline Adherencia al consumo de multimicronutrientes & 0,689 & 0,000 \\
\hline
\end{tabular}

En la tabla 3 visualizamos que $60 \%$ de madres perciben una mala atención, mientras que un 24,3\% perciben buena atención y finalmente un $15,7 \%$ califica como una atención regular.

Tabla 3

Distribución de Factor atención de Salud según percepción de las madres con niños de 6 a 36 meses de edad del Centro de Salud Guadalupe 2018.

\begin{tabular}{lll} 
Factor atención de salud & N & $\%$ \\
Malo & 42 & 60 \\
Regular & 11 & 15,7 \\
Bueno & 17 & 24,3 \\
Total & 70 & 100 \\
\hline
\end{tabular}

La tabla 4 se muestra que $72,9 \%$ de niños es no adherente y $27,1 \%$ es adherente al consumo de multimicronutrientes. 
Tabla 4

Distribución del nivel de Adherencia en niños de 6 a 36 meses del Centro de Salud Guadalupe 2018.

\begin{tabular}{lll} 
Adherencia del consumo de micronutrientes & N & $\%$ \\
No Adherente & 51 & 72,9 \\
Adherente & 19 & 27,1 \\
Total & 70 & 100 \\
Total & 70 & 100 \\
\hline
\end{tabular}

\section{DISCUSIÓN}

En relación al primer objetivo que fue determinar la percepción de las madres donde los resultados fueron que más de la mitad de las madres es decir $60 \%$ sienten o perciben una mala atención por parte del personal que labora en el Centro de Salud Guadalupe lo cual se vio reflejado mediante la encuesta tomada, además del valor de significancia que dio $p=0.00$, esto no es beneficioso para ambas partes madre e hijo y centro de salud, siendo perjudicado principalmente el niño así mismo el personal de salud ya que al percibir o sentir una mala atención hacia la madre dará paso a que no traigan a sus hijos a los controles para brindarles todo el paquete CRED, específicamente al control de hemoglobina, ya que si no se da un tratamiento indicado habrá aumento en las coberturas de niños con anemia haciendo también un problema de salud para el Estado. Es por ello que la atención brindada es pieza clave para que se dé una buena adherencia, el hecho que los usuarios perciban una buena y cálida atención dará en primer lugar confianza hacia el profesional que brindo la atención, en este caso el usuario principal son las madres que traen a sus hijos a los controles de CRED, esto pasa a ser primordial ya que de eso depende mucho la aceptación que ellas tendrán acerca de los Multimicronutrientes.

Los resultados obtenidos guardan relación con los encontrados por Rodríguez (2016), que realizó una investigación en Chimbote Perú. Cuyo objetivo fue conocer los factores asociados con la adherencia en 3 meses a la Suplementación con Multimicronutrientes niños entre 6 y 24 meses de edad donde se encontró que factores institucionales se relacionan significativamente con la adherencia a la suplementación: el tiempo de espera $(p=0.0186)$ $y$, horario de atención ( $p=0.00014$ ), esperar más de 2 horas $(\mathrm{OR}=4.4)$, con horario de atención inadecuada $(\mathrm{OR}=4.2)$, y de mala atención recibida $(O R=2.2)$ se constituyen en riesgo elevado para una no adherencia. Asimismo Villarreal (2013), cuyos resultados evidencian que la entrega de los multimicronutrientes por el centro de salud donde se atienden, debe ser adecuado para lograr resultados positivos en el tratamiento, por otra parte pese a que se hace la entrega, no se realiza el tratamiento indicado de forma adecuada, por diversos factores, pero la más resaltante es la manera de preparación de MMN en comidas liquidas, siendo el factor principal la consejería a cargo del personal de salud en este caso la enfermera y nutricionista, porque no dan tiempo para exponer una eficaz consejería a las madres. Otra causa de que no se dé un tratamiento adecuado es la distancia de los establecimientos de salud y el personal de salud escaso, disminuyendo así la realización de las visitas domiciliarias que es fundamental, también que hay madres primerizas y menores de 26 años que no brindan los alimentos necesarios para el organismo del niño. Sin embargo, las madres que poseen experiencia son mayores de 26 años estas son las que dan aporte nutricional superior a las otras madres.

Por consiguiente, en relación al segundo objetivo el $58,6 \%$ es no adherente. Esto muestra que existe una relación significativa entre ambas variables bajo fiabilidad de la prueba estadística de Rho de Spearman, con un nivel de significancia de $p$ valor $=0,00$, esto nos indica que la mala atención de salud conlleva a que haya desistencia del consumo de Multimicronutrientes, como se muestra en el primer objetivo el factor principal, sería la mala atención que se estaría dando en el centro de salud donde se hizo el estudio, es notable que esto depende del el personal en dicho centro de salud. Por otro lado basándonos en la bibliografía UNICEF definió que la Atención de Salud como "la asistencia esencial, basada en métodos y tecnologías prácticos, científicamente fundados y socialmente aceptables, puesta al alcance de todos los individuos y familias de 
la comunidad". Concepto que se vio que no es aplicado de manera correcta por parte del personal que brinda la atención a las madres. Asimismo pueden existir factores limitantes en la interacción personal de salud y madre, siendo estos los sistemas deficientes de distribución delos micronutrientes, falta de conocimiento y adiestramiento del personal de salud, proveedores de asistencia sanitaria recargados de trabajo, falta de incentivos y retroalimentación sobre el desempeño, consultas cortas, poca capacidad del sistema para educar a los pacientes, demoras en la atención de salud, maltrato al paciente entre otros, estos factores hacen que no haya una atención como se debería dar es decir poniendo en primera instancia lugar a la calidad de atención.

Contrastando Carrión (2015) en la investigación titulada "Factores que influyen en el consumo de multimicronutrientes en niños de 6 a 35 meses, Establecimiento de Salud Acora - Puno". Cuyo objetivo fue determinar los factores que influyen en el consumo de multimicronutrientes. Donde los resultados fueron que $55.3 \%$ y $72.35 \%$ de las cuidadoras prepara y administra de manera incorrecta con relación a los factores institucionales como la frecuencia de consejería de multimicronutrientes, el $59.6 \%$ de las madres refieren que reciben consejería en todas sus citas para su control de Crecimiento y Desarrollo (CRED), de las cuales el $42.6 \%$ tienen niños que ingieren incorrectamente; pero ninguno de los niños consumen los multimicronutrientes correctamente y sus madres reciben consejería solo en algunos controles del CRED, por premura de tiempo del personal de salud. Por otro lado $100 \%$ de madres nunca han recibió visitas domiciliarias con motivo de seguimiento, supervisión del consumo de Multimicronutrientes. Por otro lado con relación hacia la aceptación y/o Adherencia, un $76.6 \%$ de niños no aceptan como debería de ser los multimicronutrientes, y las madres refieren que a sus hijos no les agradan los multimicronutrientes.

Finalmente, esta investigación se acepta la hipótesis alterna mediante la prueba estadística Rho de Spearman, que mediante el rango encontrado nos da a conocer que al hacer un cruce de variables y sus respectivos resultados dice que más de la mitad $(60 \%)$ de las madres perciban mala atención por parte del personal lo que conlleva a que la mayoría $(58,6 \%)$ de las madres de los niños sea no adherente haciendo una buena correlación significativa entre ambas con un valor de $p=0.00$. Haciendo este caso un problema que perjudica a toda la sociedad.

\section{CONCLUCIÓN}

Primero: En relación al objetivo general los resultados fueron estadísticamente significativas, estas fueron validadas mediante la prueba estadística de Rho de Spearman obteniendo un valor $\mathrm{P}(<5 \%)$ porcentaje aceptable, lo que indica que se acepta la hipótesis alterna, es decir que si existe correlación positiva moderada entre el factor atención de salud y la adherencia al consumo de los Multimicronutrientes con un coeficiente de relación de 0.989 en madres con niños de 6 a 36 meses, Centro de Salud Guadalupe.

Segundo: La mayoría de las madres con niños de 6 a 36 meses perciben una mala atención por parte del personal de salud del Centro de Salud Guadalupe, lo cual hace que la relación sea significativa.

Tercero: La mayoría es no adherente al consumo de multimicronutrientes en madres con niños de 6 a 36 meses, que acuden al Centro de Salud Guadalupe, lo cual hace que la relación sea significativa.

Declaración de financiamiento y de conflictos de interés

El estudio fue financiado por los autores, quienes declaran no tener algún tipo de conflicto de interés en la investigación realizada

\section{Correspondencia:}

Mayumy Mamani Tito

Correo electrónico:

mayumy.95@gmail.com

Yovana Jarata Hancco

Correo electrónico:

yovijh@gmail.com 


\section{REFERENCIAS BIBLIOGRÁFICAS}

OMS. (2003). Adherencia al tratamiento. Recuperado de https://www.who.int/mediacentre/news/ releases/2003/pr54/es/

Mendoza,O. (2016). Norma Técnica de calidad de atención. Ministerio de Salud. Recuperado de http://cmp.org.pe/wpcontent/ uploads/2016/07/GTIndicadoresCalidad/ EstandaresCalidadSaludC MP2016.pdf

Huerta, E. (2014). Tiempo de atención en Hospitales del Perú bajo 70 a 15 minutos. Recuperado el 19 de Abril de 2018, de http://vital.rpp.pe/expertos/ minsa-tiempo-de-atencion-en-hospitalesdelperu-bajo-de-70-a-15-minutos-noticia-658973

Lazarte, A. (2017). Factores relacionados a la no adherencia del consumo de multimicronutrientes chispitas en madres de niños de 6 a 36 meses, usuarios del Centro de Salud Carlos Showing Ferrari, Amarilis-2016. (Tesis para maestrìa, Universidad de Huánuco). Recuperado de http://repositorio.udh.edu.pe/ bitstream/handle/123456789/316/OKK\%20 TESIS $\% 2$ OVERSIONAL \% 2 OFINAL \% 20 ANA \% 20 L AZARTE \% 20 MAESTRIA. pdf?sequence=1\&isAllowed $=y$
Rodriguez, J. (2016). Factores asociados con la adherencia en 3 meses a la Suplementación con Multimicronutrientes en niños entre 6 y 24 meses de edad, Chimbote, 2016. (Tesis de licenciatura, Universidad Nacional de Santa Chimbote). Recuperado de http://repositorio.uns.edu.pe/ bitstream/handle/UNS/2909/46292.pdf?sequen $\mathrm{ce}=1$ \&isAllowed $=\mathrm{y}$

Villarreal, I., Cala, E., Cortéz, J. (2013). Percepción de madres de niños de 6 a 23 meses de edad respectivo al consumo de micronutrientes "chispitas Nutricionales" en el Municipio de Puerto Carabuco - La Paz 2012. (Tesis de maestría, Universidad Mayor de San Andrés)

https://repositorio.umsa.bo/handle/123456789/16273

Carrión, D. (2015). Factores que influyen en el consumo de multimicronutrientes, en niños (as) de 6 a 35 meses, establecimiento de salud acora i -4, Puno 2014.(Tesis de licenciatura, Universidad Nacional del altiplano). Recuperado de http:// repositorio.unap.edu.pe/handle/UNAP/1887

Recibido: 5 de junio Aceptado: 15 de setiembre 